\title{
CARACTÉRISTIQUES DIFFÉRENTIELLES DU TISSU CONJONCTIF DES BOVINS NORMAUX ET “ CULARDS "
}

\author{
R. Boccard. - Station de Recherches sur la Viande, C.N.R.Z., \\ 63-Theix par St-Genès-Champanelle.
}

B.-L. Dumont, O. SchмтTт. - Laboratoive de Recherches sur la viande, C.N.R.Z., 78-Jouy-en-Josas.

Les principales caractéristiques du tissu conjonctif (importance, répartition, solubilité) ont été étudiées sur cinq muscles (Psoas major, Longissimus dorsi, Semi tendinosus, Pectoralis profundus et Triceps Brachii laterale) de châtrons charolais normaux et culs-de-poulain (ou "culards ") de 3 ans. Les deux types d'animaux ont présenté des différences pour toutes les caractéristiques considérées. Les culards ont une moindre teneur en tissu conjonctif. Exprimées en ug par $g$ de matière sèche, les quantités moyennes d'hydroxyproline furent, pour les 5 muscles, respectivement de 1267, 2294, 2884, 3426 et $3981 \mathrm{chez}$ les animaux culs-de-poulains et de 1925, $2669,4825,5645$ et 6140 chez les sujets normaux. Examinés sur des coupes histologiques de grande surface, la trame de tissu conjonctif se présente comme plus lâche et délimitant des myoskhènes de plus grande taille chez les sujets culards. La solubilité du collagène est plus grande chez les culards où les pourcentages de collagène solubilisé dans les 5 muscles (après cuisson de broyats pendant 6 heures à $90^{\circ} \mathrm{C}$ ) ont été respectivement de $22,93,22,43,17,20,16,55$ et 17,35 p. 100 alors qu'ils n'étaient, chez les normaux, que de $21,58,19,78,15,98,13,78$ et 15,13 p. 100 . De ces particularités du tissu conjonctif, il est résulté des différences dans l'indice de dureté, mesuré au Warner-Bratzler, systématiquement plus faible chez les culards que chez les normaux $(3,18$, $4,57,10,21,8,33$ et 10,43 pour les 5 muscles des culs-de-poulain contre $5,73,6,46,15,86,19,01$ et 18,13 ).

La différence dans la répartition et dans l'aspect de la trame du tissu conjonctif pourrait être, de même que celle intéressant la teneur, un trait essentiel au niveau du muscle, de la manifestation du caractère "culard ". Cet aspect particulier du conjonctif du culard pourrait être utilisé comme critère distinctif de ce type d'animal. D'autre part, bien que cette différence de répartition du tissu conjonctif puisse expliquer les différences enregistrées dans sa solubilité, il n'est pas exclu de penser que la plus grande solubilité du collagène des culards pourrait résulter également d'une différence de maturité physiologique, moindre chez les culards.

\section{STRUCTURE MUSCULAIRE COMPARÉE DE PORCS LARGE-WHITE E'T PIETRAIN}

\author{
B.-L. Dumont, O. Schmitr. - Laboratoire de Recherches sur la Viande, C.N.R.Z., \\ 78-Jouy-en-Josas.
}

Par rapport à ceux de sujets comparables (même sexe, même poids total de la musculature) de race Large-White, les muscles des porcs Piétrain peuvent être soit proportionnellement plus lourds et, au niveau de leur section transversale médiane, plus volumineux (Semi membranosus), soit simplement plus volumineux (Pectoralis profundus ou Brachialis), soit plus légers mais de volume équivalent (Triceps brachii caput laterale). La structure de ces différentes catégories de muscles a été étudiée en utilisant une méthode d'analyse (O. Schmirt et B.-L. Dumont, Ann. Biol. anim. Bioch. Biophys., 1969, 9, 123-134) reposant sur l'examen de coupes minces $(10 \mu)$ de la totalité du muscle. Dans tous les muscles hypertrophiés en volume (ou en volume et en masse) l'importance du conjonctif interne est plus faible, alors que dans le cas du Triceps brachii caput 